\title{
CaloCube: a new homogeneous calorimeter with high-granularity for precise measurements of high-energy cosmic rays in space
}

\author{
Gabriele Bigongiari* on behalf of the CaloCube collaboration \\ INFN Pisa, Largo Bruno Pontecorvo 3, I-56127 Pisa, Italy \\ gabriele.bigongiariepi.infn.it
}

\begin{abstract}
The direct observation of high-energy cosmic rays, up to the $\mathrm{PeV}$ region, will depend on highly performing calorimeters, and the physics performance will be primarily determined by their acceptance and energy resolution. Thus, it is fundamental to optimize their geometrical design, granularity, and absorption depth, with respect to the total mass of the apparatus, probably the most important constraints for a space mission. Furthermore, a calorimeter based space experiment can provide not only flux measurements but also energy spectra and particle identification to overcome some of the limitations of ground-based experiments. CaloCube is a homogeneous calorimeter whose basic geometry is cubic and isotropic, so as to detect particles arriving from every direction in space, thus maximizing the acceptance; granularity is obtained by filling the cubic volume with small cubic scintillating crystals. A prototype, instrumented with CsI(Tl) cubic crystals, has been constructed and tested with particle beams.
\end{abstract}

EPS-HEP 2017, European Physical Society conference on High Energy Physics 5-12 July 2017

Venice, Italy

\footnotetext{
* Speaker.
} 


\section{Introduction}

The direct measurement of cosmic ray (CR) spectrum in the PeV region is one of the instrumental challenge for the future $\mathrm{CR}$ experiments. Indirect measurements on ground show, around this energy region, a sudden steeping in the inclusive spectrum of particles and a progressively heavier composition, a feature known as the CR knee. So a precise knowledge of particle spectra and composition in this spectral region would allow to address fundamental issues in the field of high-energy $\mathrm{CR}$ physics. The direct $\mathrm{CR}$ detection can permit unambiguous elemental identification and a more precise energy measurement, but suffers from low exposure due to the steepness of the CR spectrum. This limitation prevented the past experiments to go beyond $100 \mathrm{TeV} / \mathrm{n}$ for the nuclei and $1 \mathrm{TeV}$ for electron+positron spectra. Direct measurements of cosmic ray proton and nuclei spectra up to $1 \mathrm{PeV} / \mathrm{n}$ and electron spectrum above $1 \mathrm{TeV}$ require an acceptance of few $\mathrm{m}^{2}$ str, an energy resolution better than $40 \%$ for nuclei and $2 \%$ for electrons, a good charge identification and a high electron proton/rejection power (at least $10^{5}$ ).
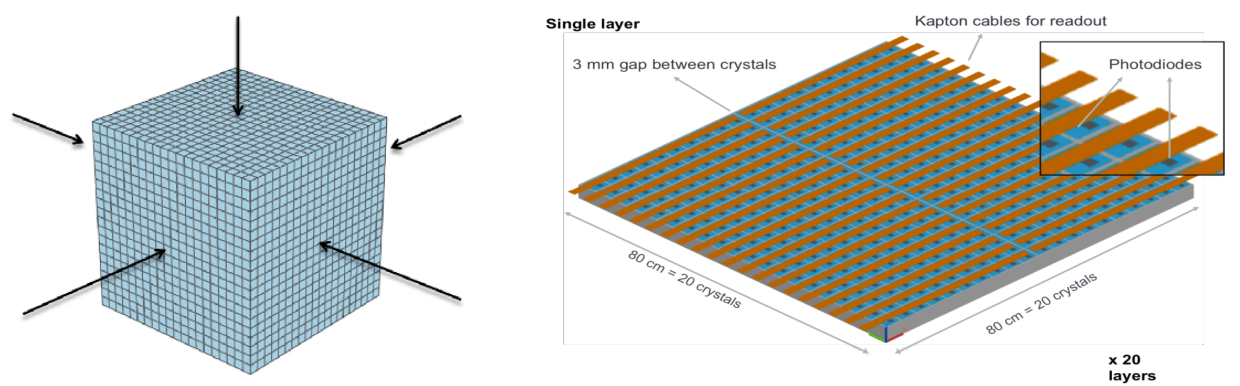

Figure 1: the CaloCube conceptual design of the 3-D highly-segmented calorimeter. The left panel depicts the complete cubic detector, while in the right panel some details about a single plane are shown.

To achieve these performances, the major constraint comes from the limitation in weight for the detectors (few tons), which severely affects both the geometrical factor and the energy resolution. The R\&D project CaloCube aims to optimize the design of a space-borne calorimeter to extend the range of direct $\mathrm{CR}$ measurements up to the $\mathrm{PeV}$ region in order to measure the knee of the lightest components [1]. The proposed solution consists of a segmented calorimeter made of a large number of cubic scintillating crystals, readout by photodiodes (PDs), arranged to form a cube (see figure 1). The cubic geometry and the homogeneity provides the possibility to collect particles from either the top or the lateral faces, thus allowing to maximize the geometrical acceptance for a fixed mass budget. The active material provides good energy resolution, while the high granularity allows shower imaging and provides criteria for both leakage correction and $\mathrm{h} / \mathrm{e}$ separation [2].

\section{The CaloCube detector}

As a starting point for the optimization of the design of the detector, a FLUKA-based model of the calorimeter has been developed, in order to evaluate the expected performances and to optimize the design. A comparative study of different scintillating crystals has been done, among CsI(Tl), $\mathrm{BaF} 2, \mathrm{YAP}(\mathrm{Yb}), \mathrm{BGO}$ and $\mathrm{LYSO}(\mathrm{Ce})$. For the hadron detection, the best choice is dictated by the balance between size (density of the absorber) and shower containment (interaction length), which 
determine energy resolution. The geometric parameters have been defined by assuming about 2 tons of active material in total; the size of the single cube has been fixed to one Moliere radius and the gap among adjacent elements has been rescaled to obtain the same active volume fraction $(\sim 78 \%)$. The signal induced in the PDs by the scintillation light has been evaluated by accounting for the light yield of the scintillators, the light collection efficiency on one face, the size and the quantum efficiency of the PD at the emission peak. Direct ionization on the PD has been also considered. Isotropic fluxes of protons hitting one face of the calorimeter have been generated and the effective geometrical factor evaluated. All the five geometries satisfied the basic requirements, by providing an effective geometrical factor of at least $2.5 \mathrm{~m}^{2} \mathrm{sr}$ with an energy resolution better than $40 \%$. Further details about the MonteCarlo studies can be found in ref. [3].
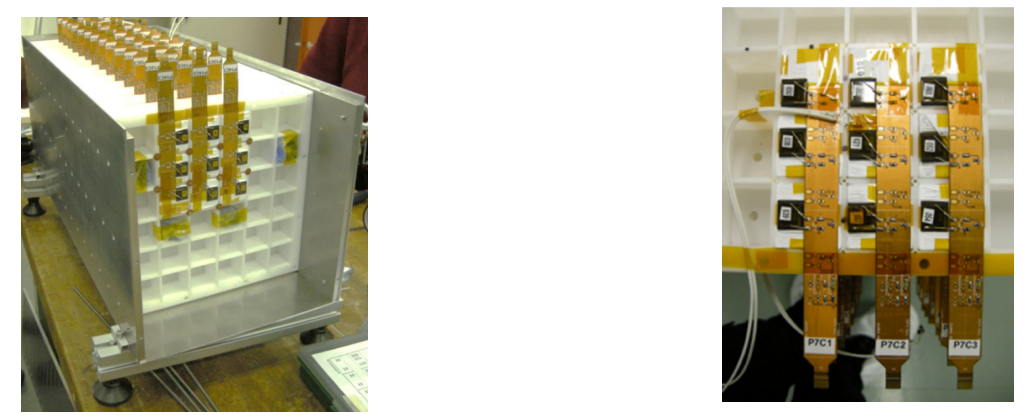

Figure 2: Left panel: picture of the first CaloCube prototype. Right panel: detail of a single layer, showing the 9 large area photodiodes placed on the crystals, that are wrapped in white Teflon tape, and the kapton cables used to read-out the signals.

To test the CaloCube concept, a prototype with $135 \mathrm{CsI}(\mathrm{Tl}$ doped) cubic crystals of $3.6 \mathrm{~cm}$ size, arranged in 15 planes of $3 \times 3$ cubes each, with a gap of $0.4 \mathrm{~cm}$ between them, has been constructed (see fig.2). CsI(Tl) has been chosen for practical reasons; it is widely available on the market at an affordable price; it has a very high light yield and its emission spectrum matches very well the spectral response of a large variety of Si-photodiodes. This prototype results to have a lateral shower containment of about 1.5 Moliere radius and a total depth of 1.35 interaction lengths, corresponding to 28.4 radiation lengths. Signals are readout by means of polyimide flexible printed circuit boards and routed to the front-end board, placed on the side of the calorimeter. The frontend electronics is based on a high dynamic-range, low-noise ASIC, developed by members of the CaloCube collaboration. The chosen PD is a large-area $\left(\sim 85 \mathrm{~mm}^{2}\right)$ sensor that, coupled to CsI(Tl) crystals and readout electronics, allows to clearly detect minimum-ionizing protons with a signalto-noise ratio of about 15 . One of the most challenging requirements is the very large dynamic range $\left(10^{7}\right)$ ranging from $20 \mathrm{MeV}$ for minimum ionizing protons to $10 \%$ of the energy of a $\mathrm{PeV}$ proton. This will be accomplished by using also a second small area PD $\left(\sim 1.6 \mathrm{~mm}^{2}\right)$.

\section{Tests with particle beams}

The first version of the prototype was tested at CERN with different particle beams (see table 1). In 2013 and 2015 it was exposed to ion beams extracted from the H8 line of CERN SPS. The beam contained $\mathrm{A} / \mathrm{Z}=2$ fragments produced by a primary $(\mathrm{Pb} / \mathrm{Ar})$ beam colliding with a target (Be/Poly). The experimental set-up included a Silicon tracking system in front of the calorimeter to provide particle trajectory information and $\mathrm{Z}$ tagging. The single-crystal performances were 

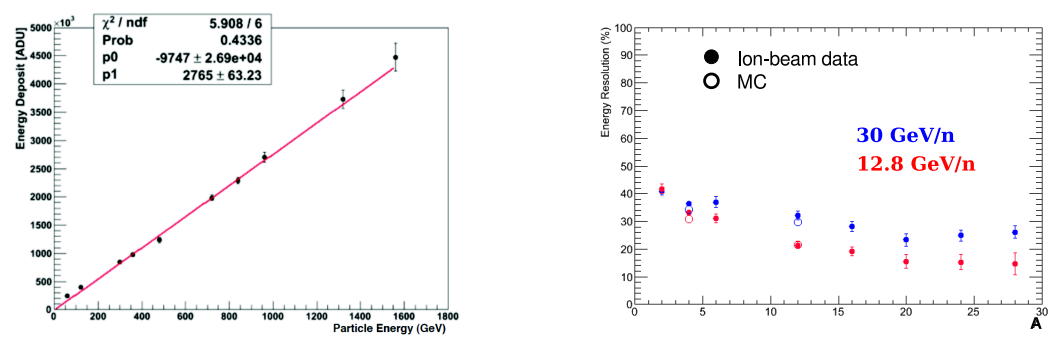

Figure 3: Left panel: preliminary result showing the linearity of the response of the prototype to the 30 $\mathrm{GeV} / \mathrm{n}$ beam as a function of the energy of the various ions. D, He, B, C, O, Mg, Si, S, Ti and Fe nuclei have been selected by the external silicon tracker. Right panel: energy resolution as a function of the ion mass number and of the beam energy, for showers having the same containment.

\begin{tabular}{|c|c|c|}
\hline Test & Beam & Energy \\
\hline Feb 2013 & ions $\mathrm{Pb}+\mathrm{Be}$ & $13-30 \mathrm{GeV}$ \\
Mar 2015 & ions Ar + Poly & $19-30 \mathrm{GeV}$ \\
Aug/Sep 2015 & $\mu, \pi, \mathrm{e}$ & $50,75,150,180 \mathrm{GeV}$ \\
\hline
\end{tabular}

Table 1: Summary of beam tests.

studied by selecting non-interacting ions. The responses were equalized by normalizing to the energy deposit of non- interacting He nuclei, the most abundant fragments. The showers developing inside the calorimeter were classified on the basis of the starting point, which can determine the shower containment. An idea of the performance in a large energy release region can be obtained from the left plot of figure 3, which shows a good linearity in the response of the prototype from the low- $\mathrm{Z}\left({ }^{2} \mathrm{H}\right)$ up to the high- $\mathrm{Z}$ ions $(\mathrm{Fe})[4]$. The right panel of figure 3 shows a preliminary estimate of the energy resolution of the prototype as a function of the ion mass number of incoming interacting nuclei. Events with showers starting before of the fifth layer of the calorimeter are selected in order to ensure a good longitudinal containment. A Fluka-based model of the prototype has been developed and its predicted response is also shown in the right panel of figure 3 (open circles) in comparison with real data. A fine tuning of the MonteCarlo simulation was necessary in order to reproduce the beam-test data. In particular, an additional spread of $4.5 \%$ on the singlecrystal responses and an optical cross-talk of $14 \%$ were introduced. During the beam test at CERN in the summer 2015 , the prototype was initially exposed to $\mu$ beams to equalize the response of all the cubes that compose the calorimeter. Then an estimate of the energy resolution has been determined exposing the calorimeter to electron beams of different energy. A preliminary result is shown in the left panel of figure 4 referring to a beam of $50 \mathrm{GeV}$ electrons. The corresponding resolution is at level of $1.5 \%$, in good agreement with the expectation.

\section{Recent developments}

A new prototype has been constructed with a completely redesigned mechanics characterized by 18 layers, each equipped with a matrix of $5 \times 5$ crystals. The total depth of this new prototype is 1.6 interaction lengths, corresponding to 35 radiation lengths. In this prototype the light signal of each crystal will be read out by two photodiodes, with different sensitive area, in order to cover the full expected dynamic range. This prototype was tested in 2016 with electron and hadron beams at 

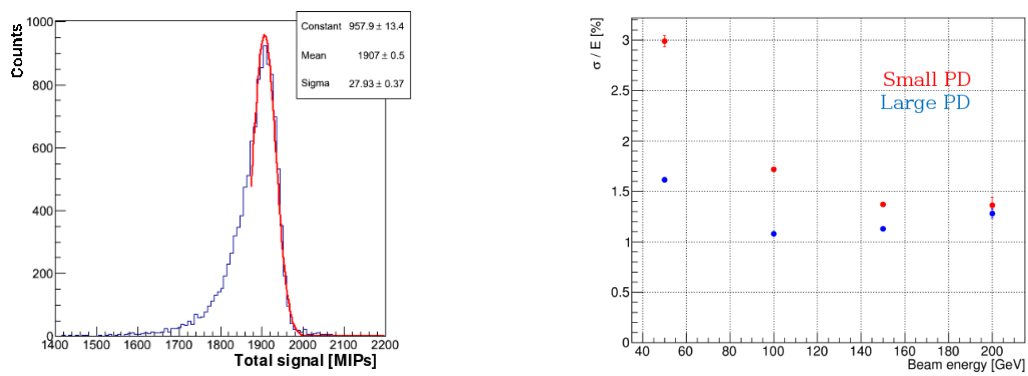

Figure 4: Left panel: measured distribution of total energy (expressed in MIP units) released with a $50 \mathrm{GeV}$ electrons beam, fit with the expected distribution (red curve). Right panel: energy resolution as a function of beam energy, obtained with large PDs (blue dots) and small PD (red dots).

the H4 line of CERN SPS. The right panel of the figure 4 shows a first glance at the performances of this improved prototype. Using the large area photodiode, the measured energy resolution for electromagnetic showers is better than $1.5 \%$ up to $200 \mathrm{GeV}$. Comparable performances above 200 $\mathrm{GeV}$ can be obtained also using small area photodiode. The analysis of data collected during this test is currently under way.

\section{Conclusions}

An innovative calorimeter for precise measurements of high-energy cosmic rays in space is being developed by the CaloCube collaboration. The basic, a large-acceptance, deep, homogeneous and isotropic cubic detector, composed of a 3-D lattice of small CsI(Tl) crystals able to detect particles from 5 sides, has been optimized. Monte Carlo simulations show that this design can reach the required acceptance and that its granularity, coupled with the large depth, will guarantee an excellent performance in the e/p separation, as well as in the energy resolution both for electromagnetic and hadronic particles. The present results from some beam tests with two prototypes of the apparatus show good performances in the energy measurements of electron and hadronic showers: an energy resolution better than $40 \%$ for ions up to $30 \mathrm{GeV} / \mathrm{n}$ and better than $1.5 \%$ for electrons up to $200 \mathrm{GeV}$. The two-sensor readout system have been successfully tested obtaining a small-PD performances comparable with large-PD. Further beam tests are scheduled in the August and November 2017 at the CERN SPS.

\section{Acknowledgments}

This work was supported by the Istituto Nazionale di Fisica Nucleare (INFN), through the CaloCube project, and by the H2020 project AIDA-2020, GA no. 654168. The authors thank CERN for the allocation of beam time at the North Area test facility.

\section{References}

[1] Bongi M. et al., J. Phys.: Conf. Ser., 587 (2015) 012029.

[2] D'Alessandro R. et al., Nucl. Instrum. Meth., A824 (2016), pp. 609-613.

[3] Mori N. et al., Nucl. Instrum. Meth., A732 (2013), pp. 311-315.

[4] Vannuccini E. et al., Nucl. Instrum. Meth, 845 (2017), pp. 421-424. 